\title{
Storage QoS Control with Adaptive I/O Deadline Assignment and Slack-Stealing EDF
}

\author{
Young Jin Nam and Chanik Park ${ }^{\dagger}$ \\ School of Computer and Information Technology, \\ Daegu University, \\ Kyungbuk, Republic of Korea \\ yjnam@daegu.ac.kr \\ ${ }^{\dagger}$ Department of Computer Science and Engineering/PIRL, \\ Pohang University of Science and Technology, \\ Kyungbuk, Republic of Korea \\ cipark@postech.ac.kr
}

\begin{abstract}
Storage QoS control enforces a given storage QoS requirement for each I/O request from different storage clients that share an underlying storage system. This paper proposes an efficient storage QoS control scheme that features adaptive I/O deadline assignment and slackstealing EDF scheduling. Simulation results with various I/O workloads show that the proposed scheme outperforms previous approaches in terms of response time variation, average response times, and miss ratio of the target response time.
\end{abstract}

\section{Introduction}

Embedding QoS feature into a storage system needs to define storage QoS specifications, map the storage QoS specifications (requirements) onto the underlying storage resources, and enforce the storage QoS requirements for each I/O request from different virtual disks (storage clients). This paper mainly emphasizes the storage QoS enforcement, also called the real-time QoS control (briefly QoS control). It is generally accepted that a storage system is characterized by its I/O performance; that is, the IOPS and RT relationship that depicts the variation of an average response time as a function of I/O requests per second (briefly IOPS). Thus, our QoS specification should capture this basic feature of a storage system as the first step. While the storage QoS specification in a broad sense may encompass other features of a storage system, such as data reliability, system costs, our QoS specification focuses mainly on the aspect of storage I/O performance that includes an average request size, a target response time, and a target IOPS. We define a QoS requirement of the virtual disk as a storage service required from a virtual disk in terms of QoS specification. The QoS requirement from a virtual disk $i\left(V D_{i}\right)$ is represented as $\left(S Z_{i}, I O P S_{i}^{\text {targ }}, R T_{i}^{\text {targ }}\right)$, where $S Z_{i}$ represents an average I/O request size(KB), IOP $S_{i}^{\text {targ }}$ represents a target IOPS, and $R T_{i}^{\text {targ }}$ represents a target response time $(\mathrm{msec})$ [1]. The QoS requirement can be easily expanded to support a storage cluster environment and a more detailed specification having multiple pairs of a target IOPS and a target response time [1].

A few QoS control schemes for storage resources have been introduced [1, 2, 3, 5, We can categorize the characteristics of the previous schemes into three classes. Class 1 
includes the derivatives of packet-based fair queuing schemes for network resources 3 . It proportionates the entire storage bandwidth according to a given set of resource weights allotted to each virtual disk that shares the same storage system. In addition, it attempts to reorder a given I/O sequence in order to reduce overhead caused by disk head movements. Note that they do not directly take control of the demanded response time; instead, they control only the storage bandwidth. Class 2 operates mainly on a rate-based QoS control using a leaky bucket [5]. It attempts to guarantee a given QoS requirement simply by throttling the IOPS of the incoming I/O requests from each virtual disk. Class 2 is expected to have the same drawbacks as Class 1. Class 3 guarantees the target response time by assigning a deadline time to each incoming I/O request only if the current IOPS is not greater than its target IOPS and then scheduling the pending I/O requests according to the EDF (Earliest Deadline First) scheme 2]. If the current IOPS is greater than its target IOPS, I/O requests have no deadline. Otherwise, the deadline is set by adding the target response time to the current time. Let us call this type of I/O deadline assignment target-IOPS-based I/O assignment. In contrast to Class 1 and 2, this approach directly controls the target response time for a given target IOPS. It can also support a QoS requirement with multiple pairs of a target IOPS and a target response time.

\section{The Proposed Scheme}

The key features of the proposed QoS control scheme include the adaptive I/O deadline assignment based on the current IOPS and the current queue depth of a virtual disk, and slack-stealing EDF scheduling that exploits any available slack between the current time and the earliest deadline time to minimize the underlying storage overhead. Note that the proposed scheme falls into Class 3 that takes control of both the target IOPS and the target $\mathrm{RT}$ for each $\mathrm{I} / \mathrm{O}$ request from different virtual disks.

Adaptive I/O Deadline Assignment. The key idea is to adaptively determine a deadline time of each I/O request according to the current IOPS and the current queue depth for each virtual disk. First, the proposed assignment scheme obtains an actual target response time denoted by act_RT $T_{i}^{t a r g}$ of $V D_{i}$ according to the current IOPS condition with respect to its target IOPS, as given in Algorithm 1 If the current IOPS is higher than its target IOPS, an I/O request is served as if it were a best-effort I/O request having no deadline. If the current IOPS is equal to the target IOPS, the actual target $\mathrm{RT}$ is the same as the original target RT. If the current IOPS is lower than its target IOPS, its actual target RT decreases in proportion to the ratio of the current IOPS to the target IOPS. Second, the proposed assignment scheme empirically measures the current average queue depth denoted by $q$ depth $h_{i}^{\text {targ }}$ for each $V D_{i}$. It computes a unit target response time denoted by unit_rt $t_{i}^{\text {targ }}$ for $a c t_{-} R T_{i}^{\text {targ }}$, as given in Algorithm 1 Note that the unit_rt ${ }_{i}^{\text {targ }}$ is meaningful only if the current IOPS is not greater than the target IOPS. Finally, the proposed assignment scheme assigns the deadline of $r_{i}^{k}$ as a function of the current position in the queue denoted by $\operatorname{qpos}_{i}^{c u r}\left(r_{i}^{k}\right)$; that is, the deadline of an I/O request increases from the queue head in a piece-wise linear manner up to its target RT by unit_rt ${ }_{i}^{\text {targ }}$. In consequence, the proposed assignment scheme can avoid delays with the processing of I/O requests from a virtual disk having a larger target RT until all the I/O requests from virtual disks having a smaller target RT are processed. 


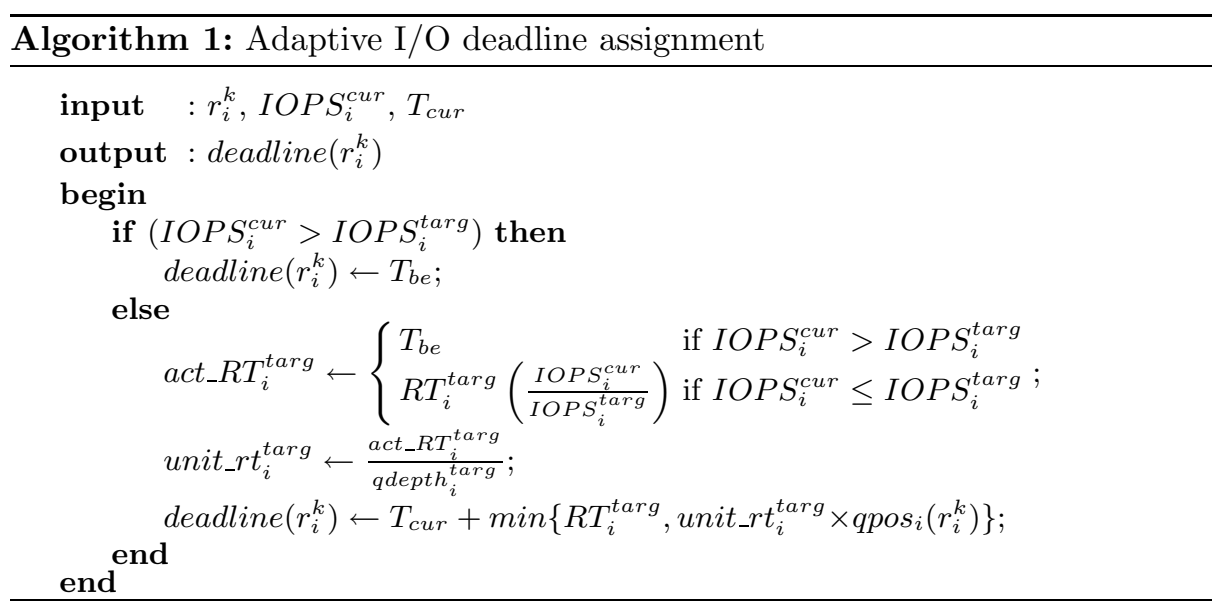

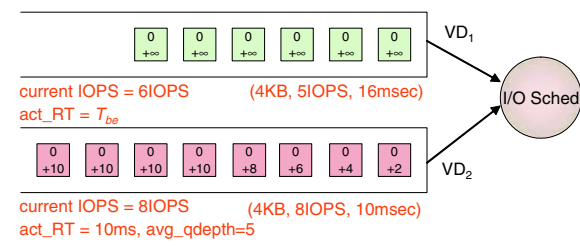

(a) heavier

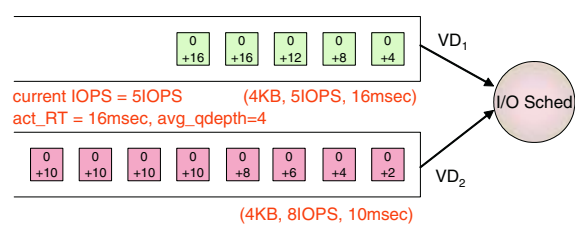

(b) equal

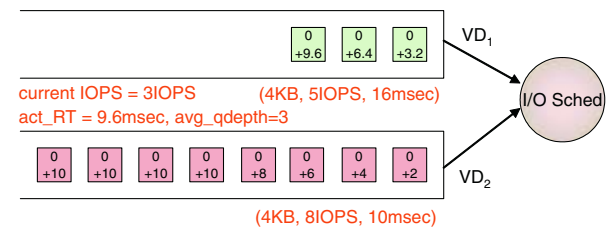

(c) lighter

Fig. 1. Examples of the adaptive I/O deadline assignment with $I O P S_{2}^{\text {cur }}=I O P S_{2}^{\text {targ }}$ : (a) heavier $\left(I O P S_{1}^{\text {cur }}>I O P S_{1}^{\text {targ }}\right)$, (b) equal $\left(I O P S_{1}^{\text {cur }}=I O P S_{1}^{\text {targ }}\right)$, (c) lighter $\left(I O P S_{1}^{\text {cur }}=I O P S_{1}^{\text {targ }}\right)$

Figure 1 show three examples of the adaptive I/O deadline assignment scheme. Each example assumes that $Q_{1}$ and $Q_{2}$ for $V D_{1}$ and $V D_{2}$ are (4KB, 5IOPS, 16msec) and $(4 \mathrm{~KB}, 8 \mathrm{IOPS}, 10 \mathrm{msec})$, respectively. First, Figure 1 presents an example for the condition that IOPS $S_{1}^{\text {cur }}>I O P S_{1}^{\text {targ }}$ and $I O P S_{2}^{\text {cur }}=I O P S_{2}^{\text {targ }}$. Since the current IOPS of $V D_{1}$ is higher than its target IOPS, the I/O deadline of each I/O request for $V D_{1}$ is set to $T_{b e}$, implying no deadline. By contrast, since the current IOPS of $V D_{2}$ is equal to its target IOPS, its actual target RT of act_RT $T_{2}^{\text {targ }}$ is $10 \mathrm{msec}$, as with its original target RT. Assuming that the observed average queue depth is 5, we obtain 
that unit_rt $_{2}^{\text {targ }}=2$ msec. Finally, the deadline of each I/O request from the queue head increases from $2 \mathrm{msec}$ up to $10 \mathrm{msec}$ by $2 \mathrm{msec}$.

Second, Figure 1(b) shows an example of the proposed deadline assignment when the current IOPS of both storage clients are the same as their target IOPS; that is, $I O P S_{1}^{\text {cur }}=I O P S_{1}^{\text {targ }}$ and $I O P S_{2}^{\text {cur }}=I O P S_{2}^{\text {targ }}$. The I/O deadline assignment of $V D_{1}$ is performed as with $V D_{2}$ in the previous example. Since the current IOPS of $V D_{1}$ is equal to its target IOPS, its actual target RT of $a c t_{-} R T_{1}^{\text {targ }}$ is $20 \mathrm{msec}$. Assuming that the observed average queue depth is 4 , we obtain that unit_rt $t_{1}^{\text {targ }}=5 \mathrm{msec}$. Finally, the deadline of each $\mathrm{I} / \mathrm{O}$ request from the queue head starts from $5 \mathrm{msec}$ and increases up to $20 \mathrm{msec}$ by $5 \mathrm{msec}$. The I/O deadline assignments by the proposed scheme for $V D_{1}$ and $V D_{2}$ in Figure 1(b) reveals that the I/O scheduler can choose the I/O requests from each queue in a fair manner. Third, Figure 1(c) presents an illustrative example for the condition that the current IOPS of $V D_{1}$ is smaller than its target IOPS. To begin, the actual target RT of act_RT targ decreases to $9.6 \mathrm{msec}$ in proportion to the ratio of the current IOPS to its target IOPS. Assuming that the observed average queue depth is 3 , we obtain that unit_rt $_{1}^{\text {targ }}=3.2 \mathrm{msec}$. Finally, the deadline of each I/O request from the queue head starts from $3.2 \mathrm{msec}$ and increases up to $9.6 \mathrm{msec}$ by $3.2 \mathrm{msec}$. Finally, observe that the proposed assignment scheme determines a deadline time of each I/O request adaptively to the changing IOPS by adjusting its target response time and by increasing the $\mathrm{I} / \mathrm{O}$ deadline by its unit target response time from the queue head. Consequently, we expect that the adaptive I/O deadline assigner will be able to give better fairness in servicing I/O requests with low RT variations in response times and provide better performance isolation to different virtual disks that share the same storage system.

Slack-Stealing EDF Scheduling: Another feature of the proposed QoS control scheme is to exploit the available slack between the current time and the earliest deadline time statistically in order to minimize storage overhead. The proposed scheduling algorithm selects an I/O request that not only minimizes the underlying storage overhead when scheduled, but also causes no deadline miss for the I/O request having the earliest deadline time. The proposed scheduling algorithm operates in two steps. First, it determines a set of $\mathrm{I} / \mathrm{O}$ requests that entail no deadline miss for the $\mathrm{I} / \mathrm{O}$ request with the earliest deadline, denoted by $R_{\text {eligible }}$. Next, it selects an I/O request that is likely to minimize the underlying storage overhead caused mainly by mechanical disk head movements. Then, the design of the proposed scheduling algorithm raises the following two issues: how to predict the service time of an I/O request and how to estimate storage overhead caused by scheduling the $\mathrm{I} / \mathrm{O}$ request.

The proposed scheduling algorithm needs to compute the service time of a given $\mathrm{I} / \mathrm{O}$ request in order to exploit or steal any existing slack time between the current time and the time when the service of the I/O request having the earliest deadline should be started to meet its deadline. Unfortunately, it is not possible to precisely predict the $\mathrm{I} / \mathrm{O}$ service time under either a disk or a storage system. Previous research to estimate the I/O service time exists based on a theoretical model of disks or storage systems [6]. However, this approach has the following drawbacks. First, it cannot capture the feature of a time-varying service time according to changes in I/O workload patterns. Second, modeling a disk or a storage system requires understanding the detailed architectures for the disk or the storage system that are generally unavailable. Thus, the proposed scheduling algorithm assumes that the I/O service time is time-variant, and it measures the time-varying I/O service time by monitoring the service time of each I/O request as used in 4]. That is, it collects the service time of each $\mathrm{I} / \mathrm{O}$ request during a given 
monitoring interval and then averages out the service times during the interval. We denote with serv_time $(t)$ the current I/O service time at time $t$. Note that a single $\mathrm{I} / \mathrm{O}$ service time is used for all virtual disks that share the underlying storage, because the service time does not include the queuing delay in the pending queues. Storage overhead of a single disk is equal to the overhead time to move from the current head position to the position to serve the I/O request. An exact computation of overhead time demands to estimate a seek time and a rotational delay between two I/O requests. However, in large-scale storage systems typically equipped with a large non-volatile cache memory, the exact estimation of storage overhead becomes almost impossible. Thus, the proposed scheduling algorithm simply estimates the overhead time between two I/O requests as the absolute distance between the start block address of the given $\mathrm{I} / \mathrm{O}$ request and that of its previous I/O request.

Algorithm 2 gives the description on the proposed scheduling algorithm. We assume that $N$ virtual disks share the same storage system. Recall that $P Q_{i}$ represents the I/O pending queue dedicated to $V D_{i}$. The notation of $r_{i}^{h}$ represents the I/O request at the head of $P Q_{i}$. Denote with $\operatorname{addr}\left(r_{i}^{k}\right)$ the start block address of $r_{i}^{k}$. We define a set of eligible I/O requests $R_{\text {eligible }}$ that resides at the head of the I/O pending queue and entails no deadline miss for the I/O request that has the earliest deadline time.

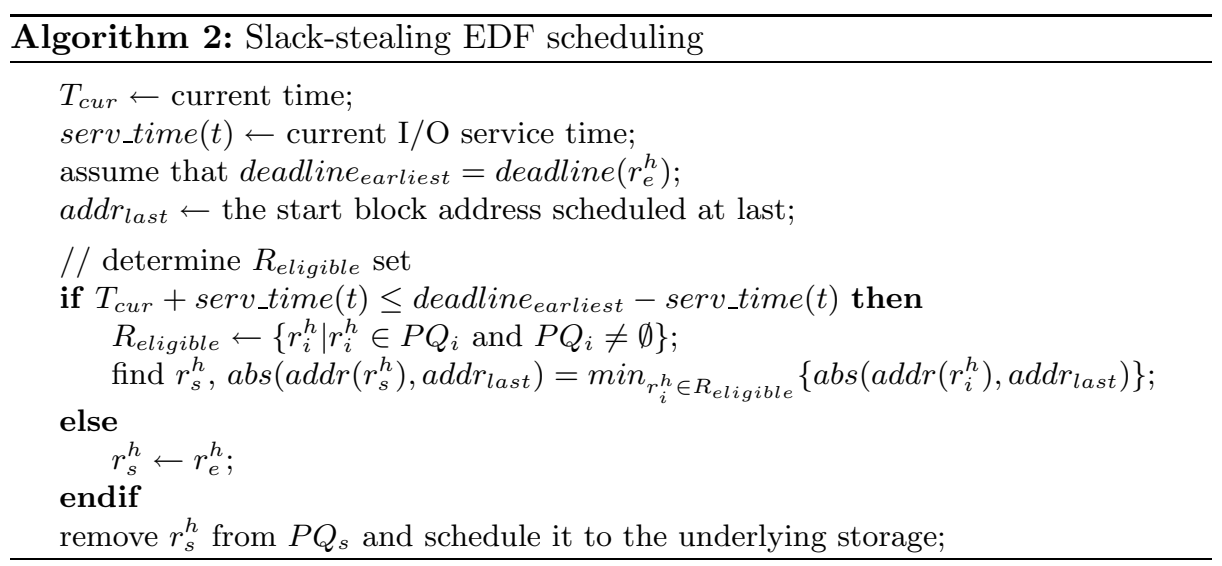

In order to determine $R_{\text {eligible }}$, the proposed scheduling algorithm examines only the first I/O request at each queue in order to minimize the I/O scheduling overhead. When the proposed scheduling algorithm inspects all the pending I/O requests within all the queues, its time complexity becomes $O(N+M)$, where $N$ is the number of the virtual disks that share the storage system and $M$ is the maximum number of I/O requests within the pending queues. Considering $M \gg N$, the approach that checks all pending I/O requests is expected to cause a considerable amount of overhead for $\mathrm{I} / \mathrm{O}$ scheduling with the increase of the number of the pending I/O requests and the number of virtual disks that share the storage system. By contrast, the time complexity of the proposed scheduling algorithm is only $O(N)$. 


\section{$3 \quad$ Performance Evaluations}

We evaluate the performance of the proposed QoS control scheme on our storage simulator that consists of an I/O workload generator, a set of virtual disks (storage clients), an underlying storage system. (See [1 for more details.) We also assume the following simulation environments. Two virtual disks named $V D_{1}$ and $V D_{2}$ issue only read I/O requests. The QoS requirement of each virtual disk is defined as $\left(S Z_{1}^{\text {targ }}, I O P S_{1}^{\text {targ }}, R T_{1}^{\text {targ }}\right)=(4 \mathrm{~KB}, 45 \mathrm{IOPS}, 70 \mathrm{msec})$ and $\left(S Z_{2}^{\text {targ }}, I O P S_{2}^{\text {targ }}\right.$, $\left.R T_{2}^{\text {targ }}\right)=(4 \mathrm{~KB}, 45 \mathrm{IOPS}, 100 \mathrm{msec})$. Note that the underlying storage system can satisfy the given QoS requirements properly. We determined the QoS requirements empirically by measuring the I/O performance of the underlying storage system for the given I/O workloads.

Adaptive I/O Deadline Assignment: To begin, we focus on evaluating the effectiveness of the proposed assignment scheme by disabling the slack-stealing EDF scheme; instead, the EDF scheduler is employed as an I/O scheduler. We use four workload sets: $W S_{1}^{d}, W S_{2}^{d}, W S_{3}^{d}$, and $W S_{4}^{d}$. Each workload set is characterized by different I/O traffic intensity from $V D_{2}$; that is, the current IOPS of $V D_{2}$ becomes higher than its target IOPS in $W S_{1}^{d}\left(I O P S_{2}^{\text {cur }}>I O P S_{2}^{\text {targ }}\right)$, the current IOPS of $V D_{2}$ is equal to its target IOPS in $W S_{2}^{d}\left(I O P S_{2}^{c u r}=I O P S_{2}^{\text {targ }}\right)$, the current IOPS of $V D_{2}$ becomes lower than its target IOPS in $W S_{3}^{d}\left(I O P S_{2}^{\text {cur }}<I O P S_{2}^{\text {targ }}\right)$, and the current IOPS of $V D_{2}$ becomes much lower than its target IOPS in $W S_{4}^{d}\left(I O P S_{2}^{\text {cur }}<<I O P S_{2}^{\text {targ }}\right)$. We can expect that the proposed assignment scheme will outperform the previous targetIOPS based I/O assignment under the workload sets of $W S_{3}^{d}$ and $W S_{4}^{d}$, where the current IOPS of $V D_{2}$ becomes smaller than its target IOPS. Table 1 compares the performance results of the target-IOPS-based I/O deadline assignment and the proposed assignment scheme. As expected, the proposed assignment scheme reduced the variation of response times of $V D_{2}$ in $W S_{3}^{d}$ and $W S_{4}^{d}$ On the contrary, it increased the variation of response times of $V D_{1}$, whereas the target RT miss ratio still remains zero. In summary, the simulations results verified that the proposed assignment scheme could overcome the drawbacks of the target IOPS-based I/O deadline assignment; that is, a high $R T$ variation of response times due to unfairness in the processing of $I / O$ requests from virtual disks having a larger target response time, and poor performance isolation by assigning its deadline based on its original target $R T$ regardless of the current IOPS.

Slack-Stealing EDF Scheduling: Recall that the proposed scheduling algorithm selects an I/O request that not only minimizes the underlying storage overhead when scheduled, but also causes no deadline miss for the I/O request having the earliest deadline time. Note that the scheduling algorithms under test will employ the adaptive I/O deadline assigner. Three workload sets, $W S_{1}^{s}, W S_{2}^{s}$, and $W S_{3}^{s}$, are used for this performance evaluation. The I/O traffic intensity for each virtual disk in $W S_{1}^{s}$ is equal to the given QoS requirements; that is, IOP $S_{1}^{\text {targ }}$ and $I O P S_{2}^{\text {targ }}$. However, the I/O traffic intensity for each virtual disk in $W S_{2}^{s}$ and $W S_{3}^{s}$ is increased by $10 \%$ and $20 \%$ respectively, compared with its target IOPS. Table 2 compares the performance results of the EDF scheduling and the proposed scheduling scheme. Observe that the proposed scheduling scheme reduced the target response time(RT) miss ratio of $V D_{1}$ and $V D_{2}$ in $W S_{2}^{s}$ and $W S_{3}^{s}$ by improving the response times of each virtual disk. To summarize, the simulation results verified that the slack-stealing EDF scheduling algorithm could reduce storage overhead by reordering the $I / O$ requests as long as the deadline times of $I / O$ requests are not missed, resulting in better target $R T$ miss ratios and average response times, compared with the EDF scheduling algorithm. 
Table 1. Summary of the performance results of the adaptive I/O deadline assignment

\begin{tabular}{|c|c|c|c|c|c|c|c|c|c|}
\hline & \multicolumn{2}{|c|}{ Avg. IOPS (IOPS) } & \multicolumn{2}{|c|}{ Avg. RT (msec) } & \multicolumn{2}{|c|}{ Var. RT } & \multicolumn{2}{|c|}{ Targ. RT miss ratio } \\
\hline & & targ-IOPS & prop & targ-IOPS & prop & targ-IOPS & prop & targ-IOPS & prop \\
\hline \multirow[t]{2}{*}{$W S_{1}^{d}$} & $V D_{1}$ & 43.5 & 43.4 & 41.4 & 40.8 & - & - & 0.21 & 0.19 \\
\hline & $V D_{2}$ & 67.7 & 68.0 & 122.1 & 118.8 & - & - & 0.58 & 0.58 \\
\hline \multirow[t]{2}{*}{$W S_{2}^{a}$} & $\overline{V D_{1}}$ & 44.8 & 44.0 & 22.8 & 22.1 & 105.0 & 73.6 & 0 & $\overline{0}$ \\
\hline & $V D_{2}$ & 44.0 & 44.7 & 26.9 & 27.5 & 159.5 & 161.4 & 0 & 0 \\
\hline \multirow[t]{2}{*}{$W S_{3}^{d}$} & $V D_{1}$ & 44.6 & 44.2 & 16.4 & 17.0 & 14.6 & 12.7 & 0 & 0 \\
\hline & $V D_{2}$ & 24.7 & 25.0 & 16.8 & 15.9 & 20.8 & 17.3 & 0 & 0 \\
\hline \multirow[t]{2}{*}{$W S_{4}^{d}$} & $V D_{1}$ & 44.5 & 44.5 & 12.4 & 12.6 & 2.2 & 2.6 & 0 & 0 \\
\hline & $V D_{2}$ & 5.0 & 5.1 & 14.0 & 12.5 & 17.9 & 6.5 & 0 & 0 \\
\hline
\end{tabular}

Table 2. Summary of the performance results of the slack-stealing I/O scheduling

\begin{tabular}{|c|c|c|r|r|r|r|r|}
\hline \multicolumn{2}{|c|}{} & \multicolumn{2}{|c|}{ Avg. IOPS (IOPS) } & \multicolumn{2}{|c|}{ Avg. RT (msec) } & \multicolumn{2}{|c|}{ Targ. RT miss ratio } \\
\cline { 2 - 8 } \multicolumn{2}{|c|}{} & EDF & prop & EDF & prop & EDF & prop \\
\hline \hline \multirow{3}{*}{$W S_{1}^{s}$} & $V D_{1}$ & 44.0 & 44.5 & 22.1 & 22.4 & 0.00 & 0.00 \\
\cline { 2 - 8 } & $V D_{2}$ & 44.7 & 44.4 & 27.5 & 23.6 & 0.00 & 0.00 \\
\hline \multirow{2}{*}{$W S_{2}^{s}$} & $V D_{1}$ & 48.7 & 49.2 & 28.2 & 28.5 & $\mathbf{0 . 0 3}$ & $\mathbf{0 . 0 2}$ \\
\cline { 2 - 8 } & $V D_{2}$ & 49.1 & 49.1 & 40.8 & 29.8 & $\mathbf{0 . 0 2}$ & $\mathbf{0 . 0 0}$ \\
\hline$W S_{3}^{s}$ & $V D_{1}$ & 53.4 & 53.8 & 45.1 & 39.3 & $\mathbf{0 . 1 6}$ & $\mathbf{0 . 0 7}$ \\
\cline { 2 - 7 } & $V D_{2}$ & 52.2 & 53.1 & 58.8 & 41.0 & $\mathbf{0 . 1 0}$ & $\mathbf{0 . 0 1}$ \\
\hline
\end{tabular}

\section{Conclusion and Future Work}

This paper proposed an efficient QoS control scheme that enforces the QoS requirements of multiple virtual disks (or storage clients) that share the same storage system. The proposed QoS control scheme consists of two key components: the adaptive I/O deadline assignment and the slack-stealing EDF scheduling for storage systems. The key of the adaptive I/O deadline assignment is to adaptively determine the deadline time of each I/O request according to the current IOPS and the current queue depth. Thus, it could overcome the drawbacks of the target IOPS-based deadline assignment: a high RT variation of response times due to unfairness in the processing of I/O requests from virtual disks having a larger target response time, and poor performance isolation by assigning its deadline based on its original target RT regardless of the current IOPS. The key of the slack-stealing EDF scheduling is to steal any available slack between the current time and the earliest deadline time in order to minimize the underlying storage overhead. The proposed scheduling algorithm selects an I/O request that minimizes the underlying storage overhead when scheduled, while causing no deadline miss for the $\mathrm{I} / \mathrm{O}$ request having the earliest deadline time. We raised two design issues concerning how to predict the $\mathrm{I} / \mathrm{O}$ service time of an $\mathrm{I} / \mathrm{O}$ request and how to estimate storage overhead (disk head movement) caused by scheduling an I/O request, and provided reasonable solutions. We implemented the proposed QoS control scheme on our storage simulator. The simulation results for the adaptive I/O deadline 
assignment under various competing I/O workload sets showed that the proposed assignment scheme not only provides better fairness with lower RT variation, but also assures a better performance isolation for each virtual disk. Performance evaluations for the slack-stealing EDF scheduling revealed that the proposed scheduling scheme could provide better target RT miss ratios and response times by reducing storage overhead under various I/O workload sets.

In future work, we plan to implement the proposed QoS control scheme on top of an actual storage system and evaluate its performance with actual I/O traffic. In addition, we need to evaluate different techniques to predict an I/O service time and storage overhead for the slack-stealing EDF scheduling.

\section{Acknowledgments}

This research was supported by the Daegu University Research Grant, No 20040825. This research has been also supported in part by the Ministry of Education of Korea for its support toward the Electrical and Computer Engineering Division at POSTECH through its BK21 program, in part by HY-SDR IT Research Center, and in part by grant No. R01-2003-000-10739-0 from the Basic Research Program of the Korea Science and Engineering Foundation.

\section{References}

1. Y. J. Nam, Dynamic Storage QoS Control for Storage Cluster and RAID Performance Enhancement Techniques. Ph.D Dissertation, POSTECH, February 2004.

2. C. Lumb, A. Merchant, and G. Alvarez, "Facade: Virtual storage devices with performance guarantees," in Proceedings of Conference on File and Storage Technologies, March 2003.

3. Y. Nam and C. Park, "A new proportional-share disk scheduling algorithm: Tradingoff I/O throughput and qos guarantees," Lecture Notes in Computer Science, vol. 1067, pp. 257-266, June 2003.

4. A. Chandra, W. Gong, and P. Shenoy, "Dynamic resource allocation for shared data centers using online measurements," in Proceedings of the 11th International Workshop on Quality of Service, June 2003.

5. H. Lee, Y. Nam, and C. Park, "Regulating I/O performance of shared storage with a control theoretical approach," in Proceedings of the 21st IEEE Mass Storage Systems Symposium/12th NASA Goddard Conference on Mass Storage Systems and Technologies (MSST2004), April 2004.

6. M. Uysal, G. Alvarez, and A. Merchant, "A modular, analytical throughput model for modern disk arrays," in Proceedings of the Ninth International Symposium on Modeling, Analysis and Simulation of Computer and Telecommunications Systems, pp. 183-192, August 2001. 